Benchmarks

\title{
Induced G1 phase arrest of fast- dividing cells improves the quality of genomic profiles generated by array-CGH
}

Georgi Manukjan ${ }^{1^{*}}$, Marcel Tauscher ${ }^{1^{*}}$, Tim Ripperger ${ }^{1}$, Adrian

Schwarzer ${ }^{2}$, Brigitte Schlegelberger ${ }^{1}$, and Doris Steinemann ${ }^{1}$

${ }^{1}$ Institute of Cell and Molecular Pathology, Hannover Medical School, Hannover, Germany, ${ }^{2}$ Institute of Experimental Hematology, Hannover Medical School, Hannover, Germany

${ }^{*}$ G.M. and M.T. contributed equally to this article.

BioTechniques 53:245-248 (October 2012) doi 10.2144/0000113938

Keywords: array-CGH; data analysis; cell culture; cell cycle arrest; proliferation

Genome-wide profiling of copy number alterations by array-based high resolution comparative genomic hybridization (array-CGH) is an important method to ensure the genomic integrity of cells in diverse conditions. We observed that the analysis of genomic profiles, in particular of fast-dividing murine leukemia cell lines, is challenging due to characteristic patterns oscillating around the array-CGH baseline. Here we show array-CGH data can be drastically improved by reducing proliferation rates of cultured cells using deprivation protocols or cell cycle inhibitors. Arresting cell cycle in the G1 phase leads to smoother genomic profiles, and hence to a more reliable detection of copy number alterations.

Array-CGH is an emerging highresolution and high-throughput molecular genetic technique enabling the detection of numerical and unbalanced genomic alterations $(1,2)$. Its relevance in distinct scientific fields is becoming more and more prominent as genomic stability needs to be proven on a high resolution level for different purposes. For instance, in induced pluripotent stem (iPS) cells (3) or genetically manipulated hematopoietic stem cells (4) it is critical to verify the genomic integrity of retrovirally transduced cells by high resolution methods to exclude genomic lesions which could have been acquired during cell manipulation and subsequent expansion.

The proliferation rate of cultured cells has a strong influence on the qualilty of genomic profiles generated by means of array-CGH. In murine $\mathrm{T}$ cell acute lymphoblastic leukemia (T-ALL) cell lines established from primary material with population doubling times of about $18 \mathrm{~h}$ (data not shown), we initially observed high oscillation around the baseline. Therefore, we asked whether the induction of G1 phase arrest by inhibition of the mammalian target of rapamycin (mTOR) pathway may improve the quality and interpretability of array-CGH data. Cells were incubated with $100 \mathrm{nM}$ mTOR inhibitor Torin 1 (R\&D Systems, WiesbadenNordenstadt, Germany) for $5 \mathrm{~h}$. The mTOR pathway integrates extracellular signals (e.g., growth factors) with intracellular conditions (nutrient availability) to regulate proliferation, cell growth, metabolism and survival (5). mTOR inhibition applying Torin 1 arrests cells in the G1 phase because of decreased translation rates of $\mathrm{mRNAs}$ involved in cell cycle progression (6).

Relative cell populations in the $\mathrm{G} 1$ phase were determined by bromodeoxyuridine (BrdU) incorporation using the APC BrdU Flow Kit (BD, Heidelberg, Germany) according to the manufacturer's recommendations, followed by flow cytometry analysis. Array-CGH was performed as previously described (7) using the Agilent Mouse Genome Microarray Kit 4x180k (Agilent Technologies, Waldbronn, Germany). For image analysis, default array-CGH settings of Feature Extraction Software (Agilent Technologies) were used. Data analysis was performed by Genomic Workbench (Agilent Technologies). As detected by gel electrophoresis and spectrophotometry, and further indicated by the derivative log ratio spread (DLRS) values below 0.2 , all analyzed DNAs were of excellent quality. All experiments have been performed twice delivering equivalent results.

A representative genomic array- $\mathrm{CGH}$ profile along with the respective $\mathrm{BrdU}$ plot of a murine cell line established from a T-ALL is shown in Figure 1A. Instead of a smooth baseline around a $\log _{2}$ ratio of zero, an oscillating signal pattern was repeatedly observed. In contrast, after applying Torin 1 the entire population could be shifted to the G0/G1 phase and the respective array-CGH profiles show a clear flat baseline using identical analysis parameters (Figure 1B).

To confirm that high proliferation rates deteriorate genomic profiles and that induction of cell cycle arrest in G1 phase using other protocols could improve array-CGH data in diverse cell systems, array-CGH was performed on the leukemia cell lines BA/F3 (murine IL-3-dependent pro-B acute lymphoblastic leukemia) and HL60 (human acute myeloid leukemia). Both cell lines display short population doubling times of about 20 and $30 \mathrm{~h}$, respectively (data not shown).

First, cell lines were kept under deprivation conditions to arrest cells in the G1 phase. For HL60, this was achieved by FCS starvation for 24 h, while G1 phase enrichment in BA/F3 cells was reached using culture medium without murine IL-3 for 24 h. Furthermore, HL60 and $\mathrm{BA} / \mathrm{F} 3$ cells were arrested in the $S$ phase by applying a synchronization protocol performing two cycles of thymidine blocking $(2 \mathrm{mM})$. Cell cycle was immediately analyzed by propidium iodide (PI) staining and flow cytometry. The 
A
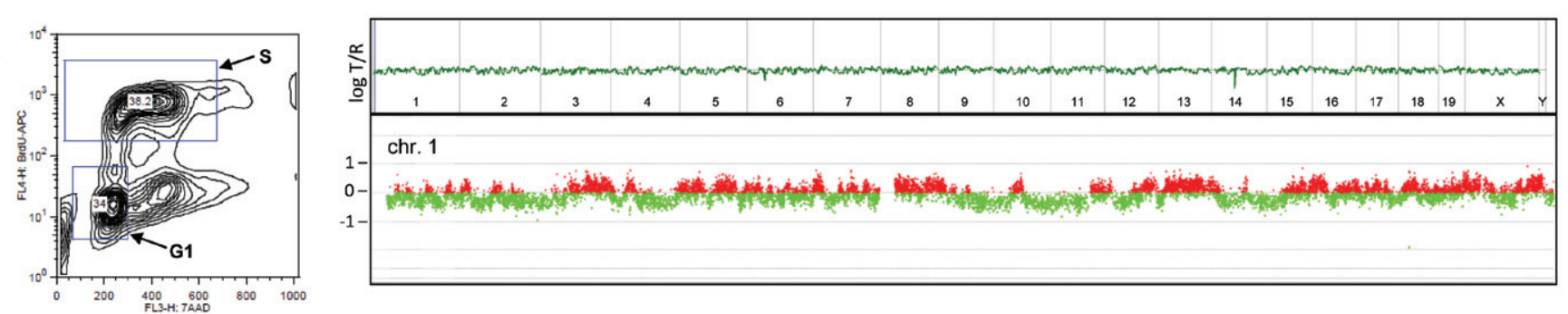

\section{B}
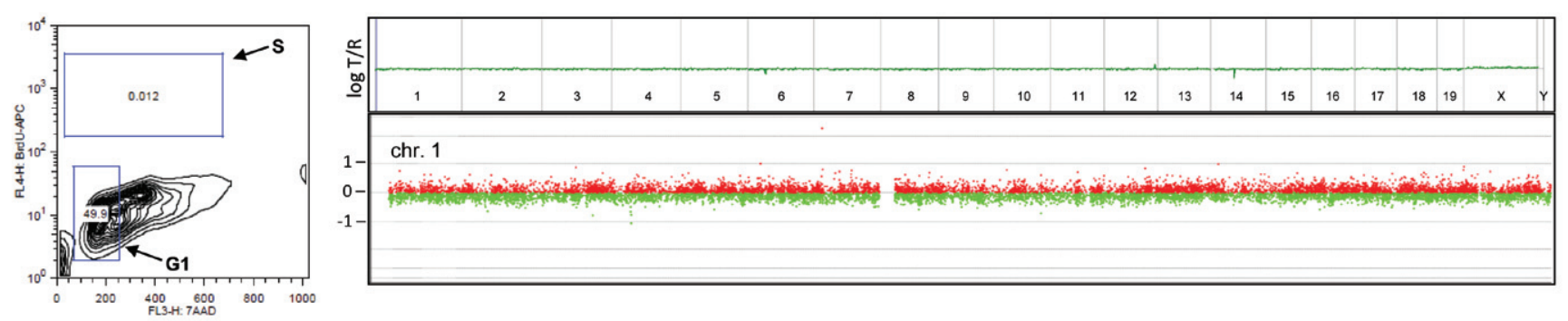

Figure 1. Flow cytometric cell cycle analysis together with respective array-CGH data of murine T-ALL cells without and after Torin1 treatment. Density plots of cell cycle analyses (left) with gated and quantified S and G1 phases, respectively, applying bromodeoxyuridine (BrdU) against 7-aminoactinomycin D (7-AAD) staining along with array-CGH profiles (right) on genomic DNA derived from a murine T-ALL cell line without (A) and after Torin1 treatment (100 nM

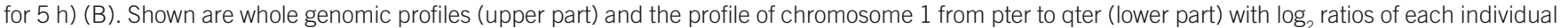
probe plotted against the chromosomal localization.

obtained data was analyzed using the FlowJo software (Tree Star Inc., Ashland, OR, USA). Array-CGH was performed as described before in duplicate with reproducible results. For HL60 cells, the Human Genome Microarray Kit 4x180k (Agilent Technologies) was used.

In $\mathrm{BA} / \mathrm{F} 3$ cells, IL3-deprivation led to a relative increase of cells in the G1 phase (90\% versus 57\%), while the $S$ phase population was nearly undetectable compared with $33 \%$ under standard culture conditions (Figures 2A1 and 2A2). Array-CGH data pattern could be dramatically improved after G1 phase arrest, as indicated by a smooth baseline even with low raw data filtering. About half of all BA/F3 cells could be arrested in the $S$ phase applying thymidine treatment (Figure 2A3). Evaluating the array-CGH baseline profiles, an oscillating pattern was detected comparable to the profiles of untreated cells (Figures 1A and Figure 2A1), making more stringent analysis parameters, i.e., increasing respective filter levels in the particular software, unavoidable to reduce background noise.

HL60 is described as a fast-dividing human myeloid cell line. Under standard culture conditions, $27 \%$ of the population resides in the $S$ phase (Figure 2B1). Relative $S$ phase could be decreased to $17 \%$ with concomitant increase of cells

\section{Signalchem Specialists in Signaling Proteins} Leader for Cell Signaling Products

\section{WWw.signalchem.com}

T: 604.232 .4600 1.866.9.KINASE F: 604.232.4601 in G1 phase by starving cells in FCS-free medium for $24 \mathrm{~h}$ (Figure 2B2). Baseline oscillation of the array- $\mathrm{CGH}$ data profile from FCS-starved cells showed no great improvement compared with normally cultured HL60 cells, which already show smooth profiles, presumably due to a $50 \%$ higher population doubling time compared with BA/F3 cells (data not shown).

After treatment of HL60 cells with thymidine, $78 \%$ of the population was arrested in the $S$ phase (Figure 2B3) that led to a remarkably increased oscillation around the baseline in arrayCGH, analogous to the observed raw data detected in untreated murine cells (Figures 1A and 2A1). Furthermore, as exemplarily shown for chromosome 1 , additional aberrations could be detected on chromosome 1q in the $S$ phasearrested population, causing putative false-positive findings.

These results demonstrate that agitated baseline patterns in array-CGH could be explained by a high proportion of cells residing in the $S$ phase, and that our observations seem to result from DNA replication during $S$ phase.

High-quality genomic profiles are required to study genomic integrity by array-CGH data with maximum sensitivity and specificity. As shown here, 

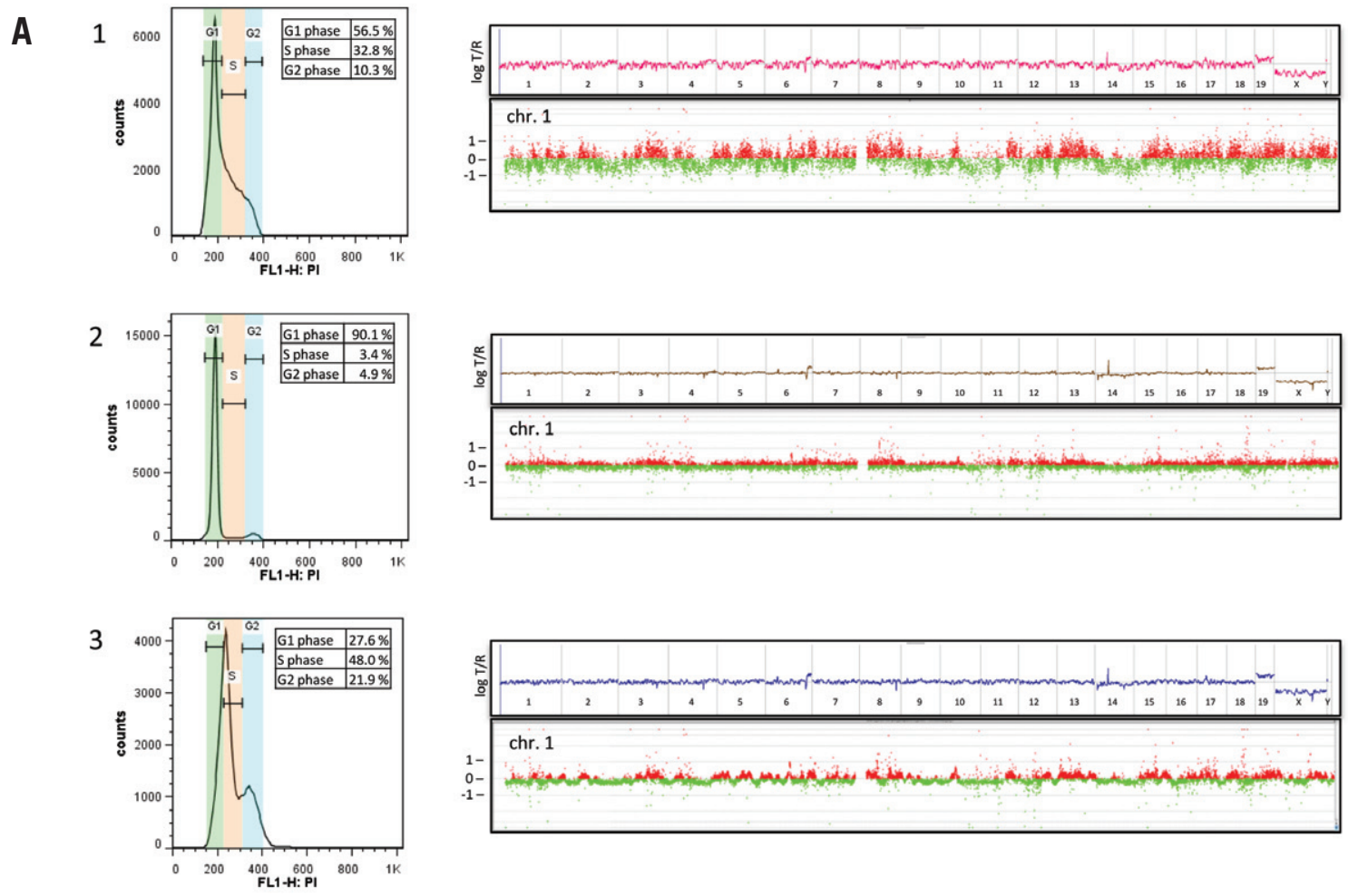

B
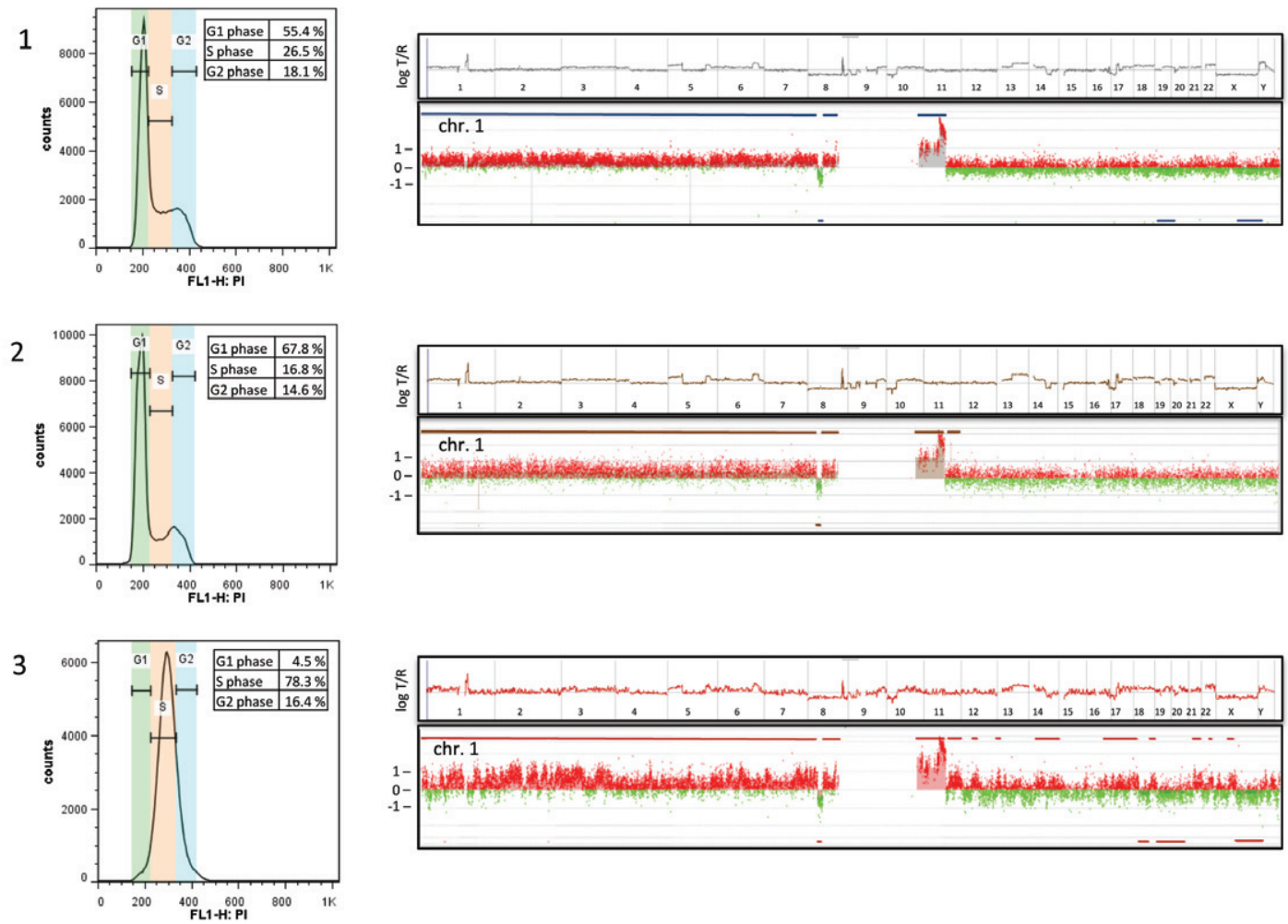

Figure 2. Flow cytometric cell cycle analysis together with respective array-CGH data of BA/F3 and HL60 cells, respectively, without and after G1 or S phase arrest. (A) Histograms of cell cycle analyses applying propidium iodide staining (left) and array-CGH profiles (right) for murine BA/F3 cells (A1) kept in standard culture conditions, (A2) in culture without murine IL3 for 24 h, (A3) after application of thymidine mediated S phase arrest. Relative cell cycle phases are marked (green: GO/G1 phase; orange: S phase; blue: G2/M phase) and quantities are indicated in tables. Whole genomic profiles as well as profiles of chromosome 1 from pter to qter are shown for each condition. (B) Histograms of cell cycle analyses applying propidium iodide staining (left) and array-CGH profiles (right) for human HL60 cells (B1) kept in recommended culture conditions, (B2) on FCS starvation for 24 h, (B3) after thymidine mediated S phase arrest. Relative cell cycle phases are marked (green: G0/G1 phase; orange: S phase; blue: G2/M phase) and quantities are indicated in tables. Whole genomic profiles as well as profiles of chromosome 1 from pter to qter are shown for each condition including marking of particular aberrations. 


\section{BioTechniques}

Monithly

Issues Now

Avaiflable

for ipad and fphorie in

the firnines

App store

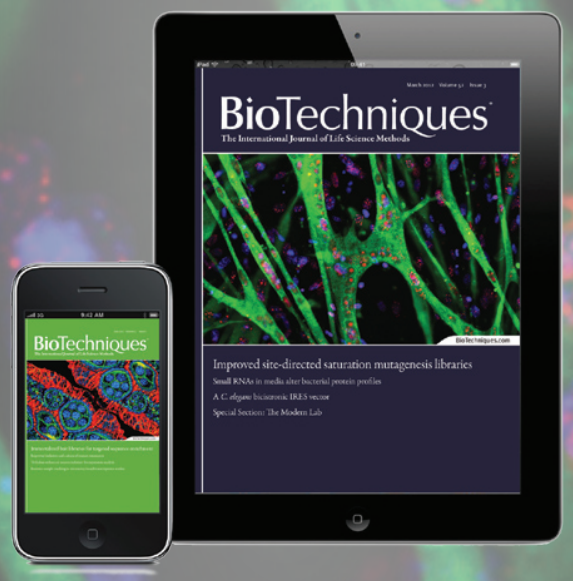

even though performing stringent quality controls at each step of array-CGH application, from DNA quality, labeling efficiency, hybridization performance, image scanning, and data extraction, results can be unsatisfactory in fastdividing cells with population doubling times of about one day or less, possibly leading to false-positive detection of copy number alterations or masking the identification of copy number alterations-e.g. those derived from subclones-because of an oscillating array-CGH baseline. Thus, we recommend monitoring population doubling times of cultured primary cells or cell lines with high proliferation rates prior to DNA extraction and suggest the induction of G1 phase cell cycle arrest in fast-dividing cells to improve the quality and facilitate the analysis of array-CGH data.

\section{Acknowledgments}

The authors would like to thank Gillian Teicke for her great support in editing the article. G.M. was supported by the Hannover Biomedical Research School (HBRS) and the $\mathrm{PhD}$ program "Molecular Medicine."

\section{Competing interests}

The authors declare no competing interests.

\section{References}

1. Pinkel, D., R. Segraves, D. Sudar, S. Clark, I. Poole, D. Kowbel, C. Collins, W.L. Kuo, et al. 1998. High resolution analysis of DNA copy number variation using comparative genomic hybridization to microarrays. Nat. Genet. 20:207-211.

2. Solinas-Toldo, S., S. Lampel, S. Stilgenbauer, J. Nickolenko, A. Benner, H. Dohner, T. Cremer, and P. Lichter. 1997. Matrix-based comparative genomic hybridization: biochips to screen for genomic imbalances. Genes Chromosomes Cancer 20:399-407.

3. Wu, G., N. Liu, I. Rittelmeyer, A.D. Sharma, M. Sgodda, H. Zaehres, M. Bleidissel, B. Greber, et al. 2011. Generation of healthy mice from gene-corrected disease-specific induced pluripotent stem cells. PLoS Biol. 9:e1001099.

4. Heckl, D., A. Schwarzer, R. Haemmerle, D. Steinemann, C. Rudolph, B. Skawran, S. Knoess, J. Krause, et al. 2012. Lentiviral vector induced insertional haploinsufficiency of ebf 1 causes murine leukemia. Mol. Ther. 20:1187-1195.

5. Laplante, M. and D.M. Sabatini. 2012. mTOR signaling in growth control and disease. Cell 149:274-293
6. Dowling, R.J., I. Topisirovic, T. Alain, M. Bidinosti, B.D. Fonseca, E. Petroulakis, X. Wang, O. Larsson, et al. 2010. mTORC1mediated cell proliferation, but not cell growth, controlled by the $4 \mathrm{E}-\mathrm{BPs}$. Science 328:1172-1176.

7. Ripperger, T., M. Tauscher, I. Praulich, B. Pabst, A. Teigler-Schlegel, A. Yeoh, G. Gohring, B. Schlegelberger, et al. 2011. Constitutional trisomy 8p11.21-q11.21 mosaicism: a germline alteration predisposing to myeloid leukaemia. Br. J. Haematol. 155:209-217.

Received 25 July 2012; accepted 12 September 2012 .

Address correspondence to Dr. Doris Steinemann, Institute of Cell and Molecular Pathology, Hannover Medical School, CarlNeuberg-Str. 1, 30625 Hannover, Germany. Email: steinemann.doris@mh-hannover.de

To purchase reprints of this article, contact: biotechniques@fosterprinting.com

\section{the App Store} and search for "BioTechniques" to download. 